\title{
Acute Branch Retinal Artery Occlusion and OCT Angiography Imaging
}

\author{
Courtney M Crawford ${ }^{1 *}$, Okezie C Igboeli ${ }^{2}$ \\ ${ }^{1}$ North Texas Retina Consultants, Fort Worth TX \\ ${ }^{2}$ Department of Ophthalmology, Carl R. Darnall Army Medical Center, Fort Hood, TX
}

Received: 12 September, 2016; Accepted: 25 September, 2016; Published: 04 October, 2016

*Corresponding author: Courtney Crawford, M.D., North Texas Retina Consultants, 101 Chuckwagon Trail, Fort Worth TX 76087, Tel: 817-441-1212; Fax: 817-441-2851; Email: courtneymcrawford@gmail.com

\begin{abstract}
A 70year old male presented with a 2day history of sudden painless decreased vision in the right eye. Other than presbyopia and senile cataracts, there was no significant past ocular history. His past medical history was significant for a cerebral vascular event of unclear origin several years prior. Evaluation revealed a branch retinal artery occlusion. Optical Coherence Tomography (OCT) angiography was employed as part of the evaluation process. We describe the OCT-angiographic findings in acute branch retinal artery occlusion.
\end{abstract}

\section{Introduction}

Retinal vascular disease is a significant cause of decreased vision and blindness. OCT has emerged as an invaluable imaging modality in the evaluation of retinal vascular diseases and is often used in guiding andassessing treatments for retinal vascular diseases.Jia $\mathrm{Y}$ et al, recently reported OCT angiography as a safe alternative to conventional fluorescein angiography. While OCT angiography has been studied since 2006, the commercial availability of angiography-enabled OCT systems began in late 2014 and these systems are in use in Europe and Asia. In this article we present the case of a patient found to have a Branch Retinal Artery Occlusion (BRAO) and the OCT angiographic findings in acute BRAO.

\section{Case Report}

A 70 year old Caucasian male presented to the New England Eye Center with a chief complaint of decreased vision in the right eye. He reported the onset, about two days prior to presentation, of sudden painless decreased in vision in the right eye which he described as a sudden appearance of a dark spot inferiorly in the right eye. His past medical history is significant for systemic hypertension and a cerebral vascular event of uncertain origin several years prior which resolved without clinical sequelae. His ocular history was significant for presbyopia, and senile cataracts in both eyes. Review of systems was significant only for hypertension. On exam visual acuity was OD 20/40+3 PH 20/25, OS 20/30-3 PH 20/20-2. Intraocular Pressure was measured OD 16 , OS 17. The patient had a visual field defect inferonasally in the right eye. There was no afferent pupillary defect and ocular motility was full. On slit lamp exam the anterior chamber was unremarkable in both eyes. Fundus exam revealed a superior temporal area of retinal whitening superior to the arcades and a Hollenhurst plaque was visualized superior to disc in the right eye. Fundus exam of the left eye revealed normal appearing macula and vessels.

Multimodal imaging techniques (i.e.fundus color photographs, OCT), were used to confirm the diagnosis of branch retinal artery occlusion (BRAO). OCT angiography was performed on the day of presentation using the prototype AngioVue OCT angiography (OCTA) system on the commercially-available Avanti SD-OCT device (Optovue, Inc, Fremont, CA), which used a SSADA software algorithm to generate OCT angiograms. The AngioVue OCTA system operated at 70,000 A-scans per second to acquire OCTA volumes consisting of $304 \times 304$ A-scans in approximately 2.6 seconds. Orthogonal registration and merging of two consecutive scan volumes were used to obtain optic disc angiography of the affected eye.

En-face sectioning was performed to visualize the various levels of ischemia; decreased perfusion was noted and can be seen superficial to the nerve head, within the peripapillary capillaries, and angio choroid (Figure 1). Increased retinal thickening corresponding to areas of ischemia is noted within the inner retinal layers (Figure 2).

Upon completion of his ophthalmic exam, the patient was admitted to the stroke service of the hospital were a complete stroke work up was completed to include echocardiography, neuro-imaging, and systemic labs all of which were unremarkable.

\section{Discussion}

Branch retinal artery occlusion is a common ocular condition. Fluorescein angiography is considered the gold standard diagnostic test, although it is often omitted in clinical care since the fundus exam is adequate for diagnosis and the test has risks, albeit low associated with it, such as allergic reaction to the flourescein dye. Acute and chronic BRAO have been described using SD-OCT. Shah, et al. described SD-OCT findings of a single 

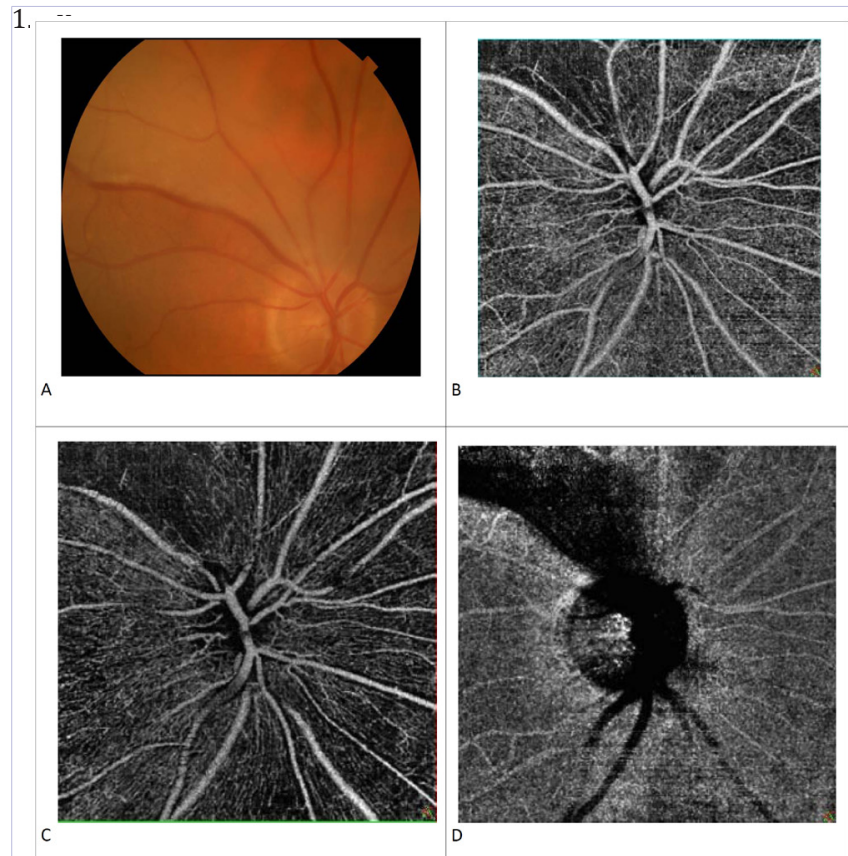

Figure 1: A. Fundus color photograph with sectoral retinal whitening along superior arcade B. Avanti angiography of superficial retina with sectoral decreased perfusion. C. Avanti angiography of deep retina with sectoral decreased perfusion. D. Avanti angiography of Choroid with decreased perfusion.

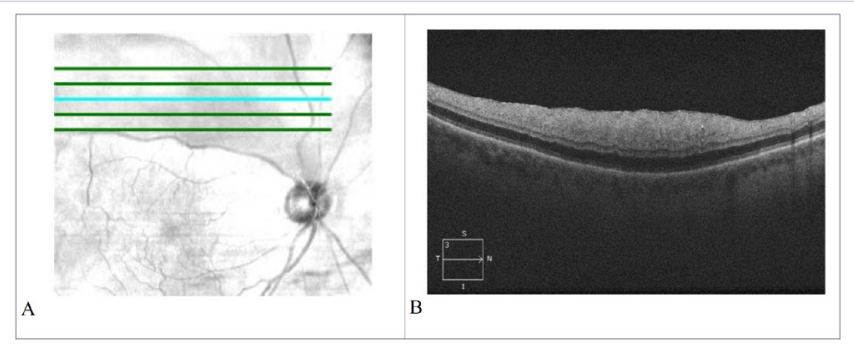

Figure 2: A. Cirrus HD 5 Line Raster. B. Cirrus HD with retinal edema of inner retina.

case of acute BRAO including hyperreflectivity and increased thickness of the inner retinal layers of the superior retina compared to the inferior retina.

Yu, et al. studied SD-OCT in 35 acute BRAO and 38 chronic BRAO. Acute findings included:

1. Thickening and hyperreflectivity of inner retinal layers, including NFL/GCL

2. Acute middle maculopathy $\mathrm{d} / \mathrm{t}$ ischemia of the intermediate and deep retinal capillary plexuses

3. Diffuse thickening and hyper reflectivity of both inner and middle retinal layers $\mathrm{d} / \mathrm{t}$ ischemia of inner and superficial, intermediate, and deep capillary plexuses.
Chronic BRAO finding include thinning and atrophy of retinal layers corresponding to acute lesions when present. Overall superficial and deep capillary ischemia occurred in $78 \%$ of eyes, isolated deep capillary ischemia or paracentral acute middle maculopathy occurred in $22 \%$.

Ahmed, et al. characterized emboli on SD-OCT in 11 cases of acute BRAO. Findings include flake of highly reflective, yet transparent material, oriented with its edge towards the direction of flow and positioned in the middle of the artery was seen in 3 cases. Reflectivity profile of the blood above and below the flake on SD-OCT and FA were normal.

This paper is the first published case report of OCT angiographic findings in acute branch retinal artery occlusion. These findings can be correlated with previously described findings on OCT. The OCT of the affected area showed thickening of the inner retinal layers consistent with previous descriptions. En face OCT angiogram of the nerve head revealed diffuse ischemia in the areas corresponding to intraluminal plaques. In the future we anticipate that OCT angiography could replace fluorescein angiography as the gold standard diagnostic test and might be more quickly and easily performed as part of routine clinical care. Future studies should directly compare OCT angiography to fluorescein angiography, enroll a large number of patients, and further examine OCT angiography findings as a potential to guide prognosis and therapy.

\section{References}

1. Ahmed Hassan J, Oliver NK, Anne Willerslev, Inger C, Michael Larsen. Embolus characterization in branch retinal artery occlusion by optical coherence tomography. Actaophthalmologica. 2014;93(1):95-96.

2. Hayreh, Sohan Singh, PA Podhajsky, MB Zimmerman. Branch retinal artery occlusion: natural history of visual outcome. Ophthalmology. 2009;116(6):1188-1194. doi:10.1016/j.ophtha.2009.01.015.

3. Karapetyan, Anushavan, Tang LS, Zeng J, Ying MD. Detection of under diagnosed concurrent branch retinal artery occlusion in a patient with central retinal vein occlusion using spectral domain optical coherence tomography. BMC ophthalmology 2014;14(1):1-5. doi:10.1186/14712415-14-91.

4. Ritter Markus, Stefan Sacu, Gábor G Deák, Karl Kircher, Ramzi G Sayegh, Christian Pruente, et al. In vivo identification of alteration of inner neurosensory layers in branch retinal artery occlusion. British Journal of Ophthalmology. 2012;96(2):201-207. doi:10.1136/ bjo.2010.198937.

5. Royster AJ, Nanda SK, Hatchell DL, Tiedeman JS, Dutton JJ, Hatchell MC. Photochemical initiation of thrombosis: fluorescein angiographic, histologic, and ultrastructural alterations in the choroid, retinal pigment epithelium, and retina. Archives of ophthalmology. 1988;106(11):1608-1614.

6. Shah Vinay A, Billi Wallace, Nelson RS. Spectral domain optical coherence tomography findings of acute branch retinal artery occlusion from calcific embolus. Indian journal of ophthalmology. 2010;58(6):523. doi:10.4103/0301-4738.71703

7. Yu Suqin, Claudine E Pang, Yuanyuan Gong, K Bailey Freund, Lawrence A Yannuzzi, Ehsan Rahimy, et al. The Spectrum of Superficial and Deep Capillary Ischemia in Retinal Artery Occlusion. American journal of ophthalmology. 2015;159(1):53-63. doi:10.1016/j.ajo.2014.09.027. 